\title{
Telemedicine and aviation
}

\begin{abstract}
The long distance flights around the world are being increase and the way how the medical situations on-board are handled must adapt this increasing. In short flights most part of these situations could be solved by a physician on-board, but himself in a non favorable place and non good conditions could result in a big problem. Telemedicine are pointed as a good tool to help a doctor, or someone trained person to use it, to solve those situations. The cost of telemedicine could be high, but the cost to change the flight plan could be worst, and in some cases could result in a late assistance.
\end{abstract}

Volume I Issue 2 - 2017

\author{
Lucas Dos Santos Boneli, Michele Dos \\ Santos Gomes Da Rosa \\ Microgravity Centre/FACA - PUCRS, Brazil
}

Correspondence: Lucas dos Santos Boneli, Microgravity

Centre/FACA - PUCRS, Porto Alegre/RS, Brazil,Tel

5551999992893,Email lucasboneli@terra.com.br

\section{Introduction}

The use of aircraft to travel short and long distance flights is spread all over the world and the knowledge around the flight environment as well. The effects of the flight have been studied since the beginning of commercial flights and nowadays are quite known and discussed among physicians and airline operators. However, the best way to handle it is still a concern and an object of study.

The term "telemedicine" appeared for the first time to help seafarers inside ships that were not carrying any doctor or paramedics onboard. ${ }^{1}$ According to the authors, this service started around 1920 and the regulations were established in 1927 during the Washington Conference on Telecommunications. It shows the importance of telemedicine in different environments, considering that a ship may take some hours to reach a port, the help of a physician even at a distance might be crucial for the patient health.

In aviation, the use of telemedicine is an increasing trend around the world, ${ }^{2}$ especially in long distance flights, however, in Brazilian aviation is a recent fact and has not been studied yet. At many cases, the call for doctors on-board the aircraft is still an aid tool for the crew and sometimes are even encouraged by the airlines companies through mileage programs, like "Doctors on Board" in Lufthansa. Nevertheless, there are some legal issues, like passengers that try to assist the doctor due to complications of care and, the most important, the unappropriated environment in which the physician is attending the passenger.

There are some tools to help the physician inside the aircraft, like on-board medical kits and AEDs (Automated External Defibrillators). ${ }^{2}$ The airplane is not carrying all the equipments that are available in a hospital and even the physician himself is in a different place and, sometimes, he is suffering from headache or other illness that might take him to evaluate the passenger's medical conditions in a non-precise way. That is why where a second opinion, coming from a remote physician, on the ground, would be powerful and valuable.

So, would the telemedicine be the best solution for an in-flight emergency? Probably the answer would be: it depends on the flight; the phase of flight; and the overflown region. If the emergency happens during the climb or the descent of the aircraft, the most reasonable action is to declare an emergency for the air traffic controller and land as soon as possible, in this case the contact with a remote physician on the ground would be a bit slow and less effective.
On the other hand, if the aircraft is stabilized at a high altitude, the descent would take a precious time and sometimes a high rate of descent might make the passenger condition worse, therefore, in this case, the help of telemedicine would be extremely valuable and more effective.

Another situation would be to imagine an aircraft flying over the ocean or in an inhabited area, where certainly there are a few number of airports, so the airport chosen to divert has to be accurately selected, which is actually a specialty of the medical centers that provide telemedicine services, once they must have a database with a lot of hospitals and their specialties.

Another concern involving directly the use of telemedicine is the cost of the service maintenance. It is known that the cost of consultations is extremely high for the companies, when is taken other cases of telemedicine uses, the fuel consumption, time spending on transportation are saved. When is taken in mind the in flight situation, these issues are similarly and also spared, like another extra fees that comes with the aircraft deviation, which turn the telemedicine a good choice.

According to a study of Rahim Valani et al. ${ }^{3}$ inside Air Canada between 2004 and 2008, the number of diversions increased when the use of telemedicine decreased. Although it could not be evaluated by the authors, certainly some of these diversions would not really be necessary. Thus, it can be affirmed that the use of telemedicine can influence the costs of operation of the airline specifically when it is about diversions due to medical problems on-board.

As pointed by Beltrame \& Maryni, ${ }^{2}$ the use of telemedicine by the airlines companies has been increasing over the past few years. MedAire, for example, a company that provides telemedicine services to airline companies, has already managed in flight emergencies from about 74 airlines worldwide. ${ }^{4}$ In Lufthansa, the use of telemedicine is increasing over the years, especially in intercontinental flights, as pointed by Weinlich et al. ${ }^{5}$ This study showed that the teleconsultation was used in 29 cases among 94 in flight medical situations in the period between 2003 and 2006.

\section{Conclusion}

The acceptance of telemedicine services is well-spread around the world, however in Brazil it is still a new and not fully comprehended "tool", considering that the first partnership between a Brazilian 
airline and a telemedicine centre was accomplished only five years ago. Case studies in this area, considering the Brazilian airlines, are scarce or even does not exist, showing a prominent area of actuation, once the number of Brazilian passengers are increasing as well the long distance flights by Brazilian airlines also.

\section{Acknowledgements}

None.

\section{Conflict of interest}

Author declares that there is no conflict of interest.

\section{References}

1. Amenta F, Dauri A, Rizzo N. Telemedicine and medical care to ships without a doctor on board. J Telemed Telecare. 1998;4(1):44-45.
2. Beltrame Francesco, Boddy K, Maryni Piergiulio. Adopting telemedicine services in the airline framework. IEEE Transactions. on Information Technology in Biomedicine. 2001;5(2):171-174.

3. Valani Rahim, Cornacchia Marisa, Kube Douglas. Flight diversions due to onboard medical emergencies on an international commercial airline. Aviat Space Environ Med. 2010;81(11):1037-1040.

4. Garrett Joan Sullivan, Alves Paulo M. Aviation Telemedicine: Past, Present and Future. 26th International Congress of The Aeronautical Science. 2008. p. 1-6.

5. Weinlich Michael, Nieuwkamp N, Stueben U, et al. Telemedical assistance for in-flight emergencies on intercontinental commercial aircraft. Journal of telemedicine and telecare. 2009;15(8):409-413. 